\title{
Treatment of Preduodenal Portal Vein
}

\author{
Mohamed Oulad Saiad, ${ }^{1,}$ El Aouni El Ouafi Kamili, ${ }^{1}$ and Najoua Aballa ${ }^{1}$ \\ ${ }^{1}$ Pediatric Surgery, Mother and Child Unit, University Hospital Mohamed VI, University Cadi Ayyad, Marrakech, Morocco \\ "Corresponding author: Mohamed Oulad Saiad, Unit 4, No. 441, Daoudiate, Marrakech, Morocco. Tel: +212-673083983, E-mail: mouladsaiad@gmail.com
}

Received 2016 February 24; Revised 2016 September 07; Accepted 2016 October 20.

Keywords: Duodenal Obstruction, Malrotation, Preduodenalportal Vein

\section{Dear Editor,}

The patient was a full-term male infant aged three days old, admitted to a secondary care center for bilious vomiting. When he was 11 days old, the pediatrician performed a barium study of the upper gastrointestinal tract that showed a right-sided stomach with signs of intestinal malrotation. At 27 days of life, he was referred to our pediatric emergency center. The patient was hypotonic and dehydrated with hypothermia. The abdomen was not distended or tender.

Abdominal and chest X-ray revealed a right sided stomach, left sided liver and a consolidation of the left lung (Figure 1A). Cardiac ultrasonography demonstrated a situs solitus and blood tests showed a positive sepsis profile; blood urea was raised, serum electrolytes were normal and blood culture was positive for extended-spectrum betalactamase producing Klebsiella. During hospitalization at our unit, non-bilious clear fluid was drained through the nasogastric tube. After ten days of rehydration and antibiotic administration, laparotomy was performed. We found a right-sided stomach and a normally right sided liver on the left, several small spleens were found on the right extremity of the liver, a quiescent intestinal malrotation with peritoneal bands from the cecum to duodenum with gallbladder and a compressive preduodenal portal vein (PDPV), which was crossing the anterior aspect and tracting the first part of the duodenum towards the left (Figure 1B). A transverse duodenotomy in the oral side ruled out an intrinsic obstruction. Ladd's procedure with lysis of adherent band and appendectomy was performed. Because of the non-bilious nasogastric aspirates during 10 days at our unit, the quiescent malrotation and the compressing aspect of the preduodenal portal vein during laparotomy, we believed that the PDPV was involved in obstruction. A gastroduodenostomy was performed. Unfortunately the baby succumbed to sepsis on postoperative day seven.

The PDPV is an extremely infrequent condition described usually as a part of polysplenia syndrome or associated anomaly in situs inversus (1). The embryogenesis of this anomaly described by Gray and Skandalakis consisted of anomalous regression of the cranial and middle anastomotic veins, which connects the two vitelline veins that run on either side of the duodenum in the embryo. The caudal anastomotic vein that runs ventral to the duodenum remains a part of the portal vein and results in the anomalous position of the vein (2).

Animal study in rats suggests autosomal recessive inheritance of the PDPV (3). Anomalies associated with PDPV include biliary atresia, preduodenal common bile duct, cardiovascular malformations and other malformations causing duodenal obstruction $(4,5)$. Because of the latter, some authors denied the role of low pressure within the PDPV in the compression of the duodenum (6). We think that in the present report not the pressure within the PDPV but its short length led to extrinsic pressure of the duodenum. However, in some cases there is a dilemma to involve the PDPV as an etiology of the duodenal obstruction when other frequent etiologies are present.

The aim of the treatment of duodenal obstruction caused by PDPV is to bypass the vein. Several procedures have been reported: an end to end duodenoduodenostomy leading to a retroduodenal position of the PDPV, gastrojejunostomy, duodenojejunostomy and a loose overbridging duodenoduodenostomy is the procedure of choice to avoid compression of the PDPVblood flow (6-8), but in our report it was impossible to perform this latest procedure with a wide loop and a gastroduodenostomy was the only way to prevent the portal vein from being compressed.

In spite of its rarity, PDPV should be considered in any infant with duodenal obstruction associated with cardiac malformation, dextrocardia and situs inversus (9). Preoperative sonography in these patients may be useful to define the position of the portal vein. Pediatric surgeons must be aware of the existence of PDPV in order to preserve its integrity from accidental damage or thrombosis with serious outcome. Preduodenal portal vein is often symptomless and usually associated with other causes of duodenal obstruction. It is particularly difficult to prove the

Copyright (c) 2016, Growth \& Development Research Center. This is an open-access article distributed under the terms of the Creative Commons Attribution-NonCommercial 4.0 International License (http://creativecommons.org/licenses/by-nc/4.0/) which permits copy and redistribute the material just in noncommercial usages, provided the original work is properly cited. 

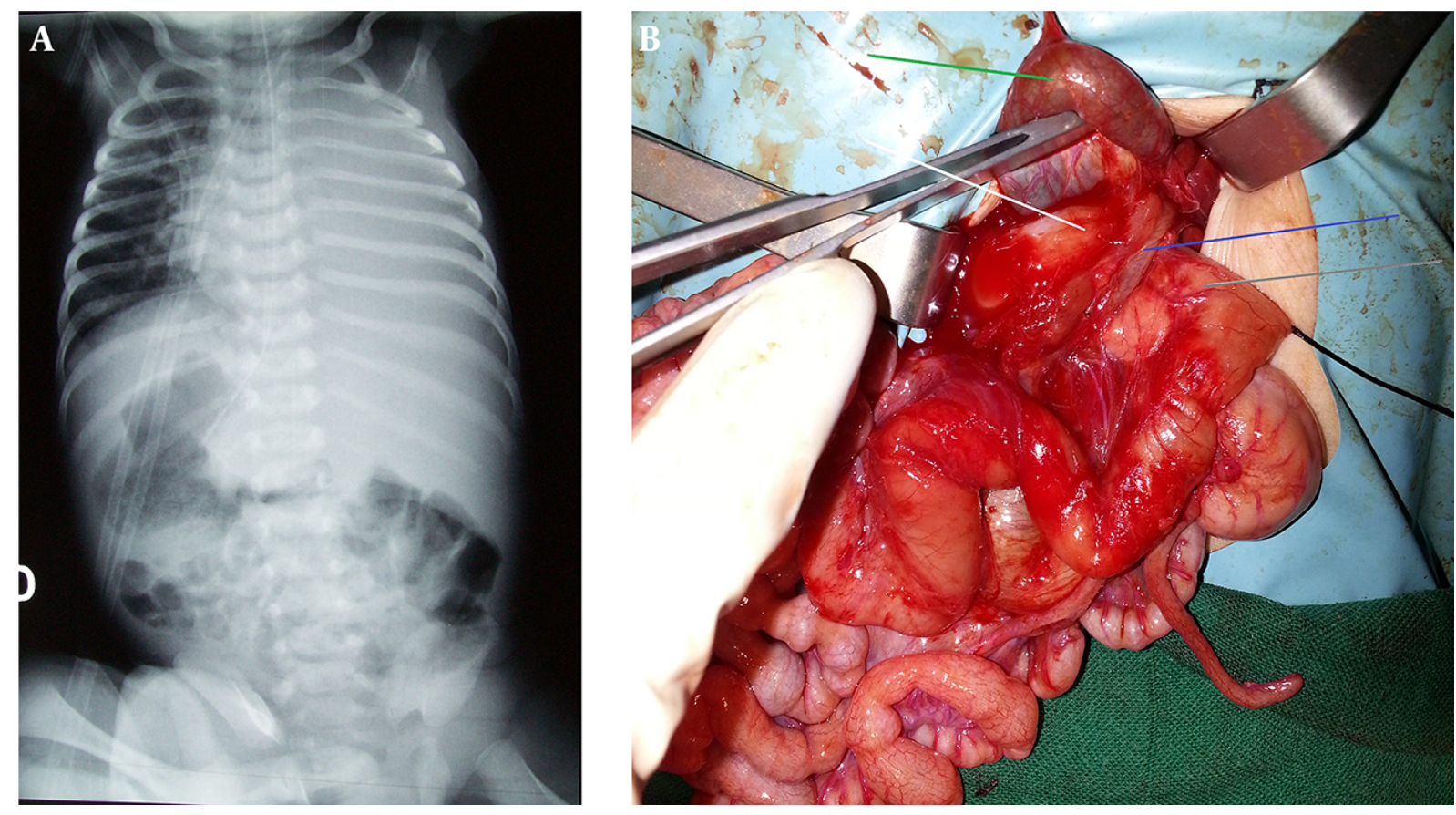

Figure 1. A, abdominal and chest X-ray showing a right-sided stomach revealed by the nasogastric tube, a left-sided liver and consolidation of the Left Lung; B, intraoperative photograph showing the gallbladder (green arrow), oral side of the duodenum (white arrow), anal side of the duodenum (gray arrow) and the short preduodenal portal vein (blue arrow) compressing and tracting the duodenum.

involvement of the PDPV in the duodenal obstruction. A careful analysis of symptoms, and surgical findings are the key factors of decision making when surgical treatment of PDPV should be considered.

\section{References}

1. Mordehai J, Cohen Z, Kurzbart E, Mares AJ. Preduodenal portal vein causing duodenal obstruction associated with situs inversus, intestinal malrotation, and polysplenia: A case report. J Pediatr Surg. 2002;37(4):5. [PubMed: 11912540].

2. Gray SW, Skandalakis JH. Embryology for surgeons. The embryological basis for treatment of congenital defects. Philadelphia: Saunders; 1972. pp. 177-8.

3. Pathak D, Sarin YK. Congenital duodenal obstruction due to a preduodenal portal vein. Indian Pediatr. 2006;73(5):423-5. [PubMed:16741329].

4. Shah OJ, Robbani I, Khuroo MS. Preduodenal portal vein with preduodenal common bile duct: an extremely rare anomaly. Am J
Surg. 2009;197(4):43-5. doi: 10.1016/j.amjsurg.2008.04.020. [PubMed: 19178906].

5. Thirumoorthi AS, Cowles RA. Preduodenal portal vein. Surgery. 2016;159(2):672-3. doi: 10.1016/j.surg.2014.08.042. [PubMed: 26395503].

6. Mboyo A, Khadir SK, Guillaume MP, Massicot R, Flurin V, Lalioui A, et al. An exceptional cause of duodenal obstruction detected antenatally: A compressive preduodenal portal vein.J Pediatric Surgery Case Reports. 2013;1(12):420-4. doi: 10.1016/j.epsc.2013.09.011.

7. Choi So, Park WH. Preduodenal portal vein: a cause of prenatally diagnosed duodenal obstruction. J Pediatr Surg. 1995;30(10):1521-2. [PubMed: 8786512].

8. Wabada S, Abubakar AM, Mustapha B, Pius S, Khalil J, Abana AK. Congenital duodenal obstruction due to duodenal atresia with preduodenal portal vein, annular pancreas, and intestinal malrotation associated with situs inversus abdominis: A case report. J Pediatric Surgery Case Reports. 2015;3(12):545-7. doi: 10.1016/j.epsc.2015.10.012.

9. D'Souza F, Nage A, Bendre P. Preduodenal Portal Vein with Situs Inversus Totalis causing Duodenal Obstruction. APSPJCase Rep. 2016;7(3):24. doi: 10.21699/ajcr.v7i3.435. [PubMed: 27398325]. 